\title{
RECONSTRUÇÃO DE IMAGENS TOMOGRÁFIACAS TIPO DICOM PELA TÉCNICA DE VETORIZAÇÃO APLICADA A FABRICAÇÃO DE PRÓTESES VIA PROTOTIPAGEM RÁPIDA
}

\section{Karilany Dantas Coutinho}

Universidade Federal do Rio Grande do Norte, Departamento de Engenharia

Biomédica, Campus Universitário, Lagoa Nova, Natal, RN, Brasil. CEP: 59072-970. karilany@ufrnet.br

Paulo Victor de Azevedo Guerra

Universidade Federal do Rio Grande do Norte, Escola de Ciência e Tecnologia, Campus

Universitário, Lagoa Nova, Natal, RN, Brasil. CEP: 59072-970.

paulovictorguerra@gmail.com

Nadyne Dayonara Maurício de Amorim

Universidade Federal do Rio Grande do Norte, Escola de Ciência e Tecnologia, Campus

Universitário, Lagoa Nova, Natal, RN, Brasil. CEP: 59072-970.

nadyneamorim@hotmail.com

Matheus André Coutinho Ferreira

Universidade Federal do Rio Grande do Norte, Escola de Ciência e Tecnologia, Campus

Universitário, Lagoa Nova, Natal, RN, Brasil. CEP: 59072-970.

matheuscoutinho10@ hotmail.com

Gustavo Kleber Bezerra Coutinho

Universidade Federal do Rio Grande do Norte, Escola de Ciência e Tecnologia, Campus

Universitário, Lagoa Nova, Natal, RN, Brasil. CEP: 59072-970. gustavo.kleber@hotmail.com

\section{Custódio Leopoldino Brito Guerra Neto}

Universidade Federal do Rio Grande do Norte, Departamento de Engenharia

Biomédica, Campus Universitário, Lagoa Nova, Natal, RN, Brasil. CEP: 59072-970. custodioguerra@yahoo.com.br

Ricardo Alexsandro de Medeiros Valentim

Universidade Federal do Rio Grande do Norte, Departamento de Engenharia

Biomédica, Campus Universitário, Lagoa Nova, Natal, RN, Brasil. CEP: 59072-970. ricardo.valentim@ufrnet.br

\section{Caroline Dantas Vilar Wanderley}

Universidade Federal do Rio Grande do Norte, Departamento de Engenharia

Biomédica, Campus Universitário, Lagoa Nova, Natal, RN, Brasil. CEP: 59072-970.

carolinevilar@gmail.com

Maria de Lourdes Silva de Arruda Morais

Liga Norte-Riograndense Contra o Câncer, Av. Miguel Castro, Dix-Sept Rosado, Natal, RN, Brasil. CEP: 59062-000.

lourd_arruda@hotmail.com

\section{RESUMO}

Atualmente é crescente a incidência de doenças malignas bem como a perspectiva de cura. No Brasil e no mundo há um grande número de mutilados em decorrência de câncer de cabeça e pescoço e câncer bucal. Com essa nova realidade, os profissionais de saúde passaram a perceber que, além da retirada do tumor e eliminação das células afetadas, tornou-se necessária também a busca pela reabilitação, na tentativa de garantir 
também a qualidade de vida do indivíduo. A Tomografia Computadorizada e Ressonância Magnética são duas técnicas comumente utilizadas para capturar informações da anatomia humana. A Prototipagem Rápida (PR) é uma técnica relativamente recente para produzir objetos com formas complexas, sendo obtida diretamente através de dados digitais tridimensionais. Este trabalho descreve um sistema que integra métodos de reconstrução tridimensional e técnicas de prototipagem rápida, possibilitando a criação de modelos médicos (biomodelos) através de dados tomográficos. Esses modelos podem ser manufaturados através de processos que são úteis em muitas aplicações médicas, tais como: auxiliar em diagnósticos, planejar de tratamentos, guiar procedimentos cirúrgicos e confecção de próteses. Os modelos fabricados via impressora 3D são construídos pela adição de camadas de material, camada por camada. A pesquisa em questão baseou-se em um estudo de um caso clínico de um paciente da Liga Norte-Riograndense Contra o Câncer (LNRCC), localizada em Natal/RN, o paciente em estudo apresentava uma lesão oncológica na região palatina esquerda. Este artigo descreve os procedimentos para a aquisição de imagens médicas no formato DICOM, a conversão desses arquivos de engenharia no formato STL e por fim a confecção da prótese via prototipagem 3D.

\title{
PALAVRAS-CHAVE:
}

Biomodelos, Prototipagem Rápida 3D, Próteses.

\section{RECONSTRUCTION OF DICOM TOMOGRAFY IMAGES BY VECTORIZATION TECHNIQUE APPLIED TO MANUFACTURING PROSTHESIS VIA RAPID PROTOTYPE}

\begin{abstract}
Currently there is a growing incidence of malignancies and the prospect of cure. In Brazil and in the world there are a great number of maimed because of head and neck cancer and oral cancer. With this new reality, health professionals began to realize that, in addition to tumor removal and disposal of the affected cells also became necessary to search for the rehabilitation, also in an attempt to ensure the quality of life of the individual. Computed tomography and Magnetic Resonance Imaging are two techniques commonly used to capture information of human anatomy. The Rapid Prototype (RP) is a relatively recent to produce objects with complex shapes technique, being obtained directly through three-dimensional digital data. This paper describes a system that integrates methods for three-dimensional reconstruction and rapid prototyping techniques, enabling the creation of medical models (biomodels) through tomographic data. These models can be manufactured using processes that are useful in many medical applications, such as: assist in diagnosis, planning treatment, guiding surgical procedures and prosthesis manufacturing. The 3D models manufactured via printer are constructed by adding layers of material, layer by layer. The research in question was based on a study of a clinical case of a patient Liga Norte-Riograndense Contra o Câncer (LNRCC), located in Natal / RN, the study patient had a malignant lesion in the left palate. This article describes the procedures for the acquisition of medical images in DICOM format, the conversion of these files in STL format engineering and finally to make the prosthesis via 3D prototyping.
\end{abstract}


KEYWORDS:

Biomodels, 3D Rapid Prototyping, Prostheses. 


\section{RECONSTRUÇÃO DE IMAGENS TOMOGRÁFIACAS TIPO DICOM PELA TÉCNICA DE VETORIZAÇÃO APLICADA A FABRICAÇÃ̃ DE PRÓTESES VIA PROTOTIPAGEM RÁPIDA}

\section{INTRODUÇÃO}

Defeitos decorrentes de cirurgia de tumor de câncer de cabeça e pescoço, traumas ou queimaduras, e defeitos congênitos são as razões mais comuns para a reabilitação maxilofacial. Dependendo da localização e do tamanho do defeito, o mesmo pode ser restaurado ou cirurgicamente ou por meio de próteses. Cirurgias reconstrutivas e microvasculares são os tratamentos preferidos para muitos pacientes, mas a disponibilidade de tecidos e alterações induzidas por radiação do tecido podem limitar os procedimentos cirúrgicos. Reconstruções cirúrgicas de defeitos faciais são tecnicamente exigentes e a reconstrução de um nariz ou uma orelha geralmente requer várias operações para alcançar um resultado esteticamente agradável, ver [15]. Nos casos em que a cirurgia reconstrutiva não é possível, uma prótese maxilofacial é utilizada como um substituto para a falta de estruturas biológicas, a fim de reabilitar a estrutura facial para restituir a aparência normal. A prótese restaura a anatomia normal, como também protege o tecido do defeito, proporcionando benefícios psicológicos para o paciente.

Deformidades ou mutilações nas regiões de cabeça e pescoço afetam o indivíduo em sua autoestima e interfere na relação interpessoal, contribuindo para o isolamento e marginalização na sociedade. De acordo com [5], as deformidades faciais são repulsivas e embaraçosas ao portador, tornando-o psicologicamente traumatizado, obrigando-o a afastar-se do convívio social. Deformidades faciais podem ocorrer também devido a traumas ou malformações congênitas. Independentemente da causa, a reabilitação protética pode ser uma opção para solucionar problemas físicos e psicológicos decorrentes da mutilação ou deformidades maxilofaciais.

Tecnologias como a captura tridimensional de superfície (digitalização 3D), CAD (Computer Aided Design) tridimensional, e os processos de fabricação de camada aditivada (ou Prototipagem Rápida e Fabricação) são na atualidade investigados em aplicações protéticas bucomaxilofaciais. No Brasil há um grande número de mutilados em decorrência de câncer de cabeça e pescoço e câncer bucal. Com essa realidade, os profissionais da área da saúde passaram a perceber que, além da retirada do tumor e eliminação das células afetadas, tornou-se necessária também a busca pela reabilitação. Desta forma, o uso da tecnologia de (PR) melhorou significativamente a concepção e fabricação de modelos complexos em uma ampla gama de aplicações. A integração de sistemas de imagens médicas com os sistemas de (PR) permite a geração de biomodelos precisos, proporcionando valiosa ajuda em áreas como planejamento cirúrgico, projeto do implante, fabricação de próteses, auxílio no diagnóstico e no planejamento do tratamento médico [4].

A Prototipagem Rápida é uma técnica relativamente nova para produzir rapidamente objetos em 3 dimensões e com formas complexas, diretamente a partir de dados computacionais [10]. Modelos sólidos são construídos por meio da adição de camadas de materiais, uma camada de cada vez, com cada camada sucessiva formando no lugar e aderindo-se à pilha de camadas previamente formadas. Imagens médicas, por exemplo, a tomografia computadorizada (TC) e a Ressonância Magnética (RM) são duas técnicas comuns para a captura de informações anatômica detalhada dos pacientes. 
Esta pesquisa consiste no estudo de técnicas para tratamento de imagens médicas para a fabricação de próteses bucomaxilofaciais, utilizando Prototipagem Rápida. O estudo é realizado desde o arquivo DICOM (Digital Imaging and Communications in Medicine) da tomografia computadorizada até a preparação para a injeção plástico na Impressora tridimensional para a confecção da prótese em estudo.

\section{MATERIAIS E MÉTODOS}

\section{Caso Clínico:}

A pesquisa baseou-se em um estudo de um caso clínico de um paciente, denominado no texto como Paciente 02 da Liga Norte-Riograndense Contra o Câncer (LNRCC), localizada em Natal/RN. O Paciente 02 em estudo, apresentava uma lesão oncológica na região palatina esquerda, ver Figura 1.

O tratamento reabilitador deste caso Clínico foi desenvolvido em duas fases, porém apenas a primeira desenvolvida neste trabalho.

A primeira fase de reabilitação foi confeccionar uma prótese pré-cirúrgica através de prototipagem rápida para ser instalada no paciente logo após a remoção da área com comprometimento oncológico. Esse dispositivo tem função de evitar a retração tecidual em decorrência da mutilação cirúrgica. Uma nova prótese intra-oral será posteriormente confeccionada pelos métodos tradicionais após 6 meses do procedimento cirúrgico realizado.

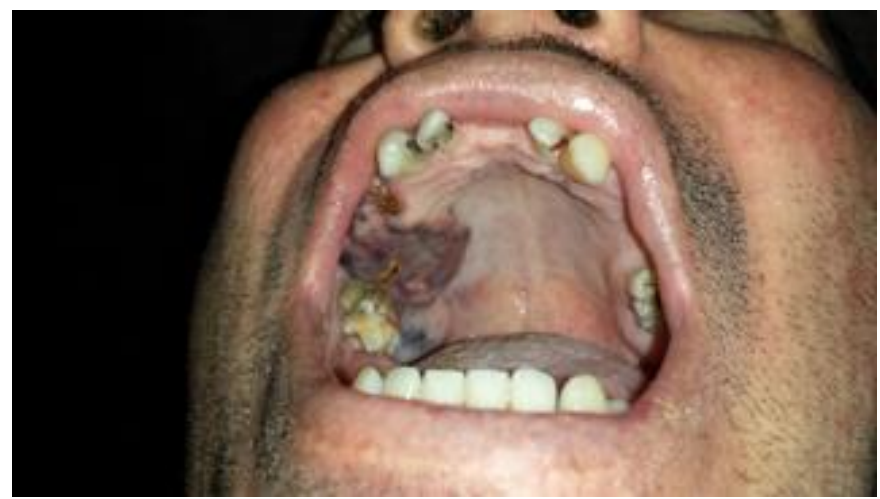

Figura 1: Caso Clínico - Paciente 02.

\section{Pré-processamento das Imagens Bidimensionais:}

Nessa etapa foi utilizado o Software Público gratuito InVesalius v.3- $\beta 5$, desenvolvido pelo parceiro da pesquisa, o CTI (Centro de Tecnologia da Informação Renato Archer, Campinas/SP, Brasil), um centro de pesquisas do Ministério da Ciência e Tecnologia.

$\mathrm{O}$ modelo 3D da região em estudo, ver Figura 2, foi concebido com base na reconstrução anatômica do Paciente 02, obtida através das imagens médicas no formato DICOM. A técnica utilizada foi a de vetorização por delineação dos contornos das regiões, baseada na diferenciação de pixéis em cada imagem axial, coronal ou sagital, conforme visto na Figura 3. A vetorização, a qual deu origem a toda a informação de reconstrução anatômica digital 3D, não só capturou a informação anatômica real como 
também todo o ruído, ou seja, regiões desnecessárias para a análise em questão. Para eliminar o efeito dos ruídos, utilizou-se o próprio software InVesalius.

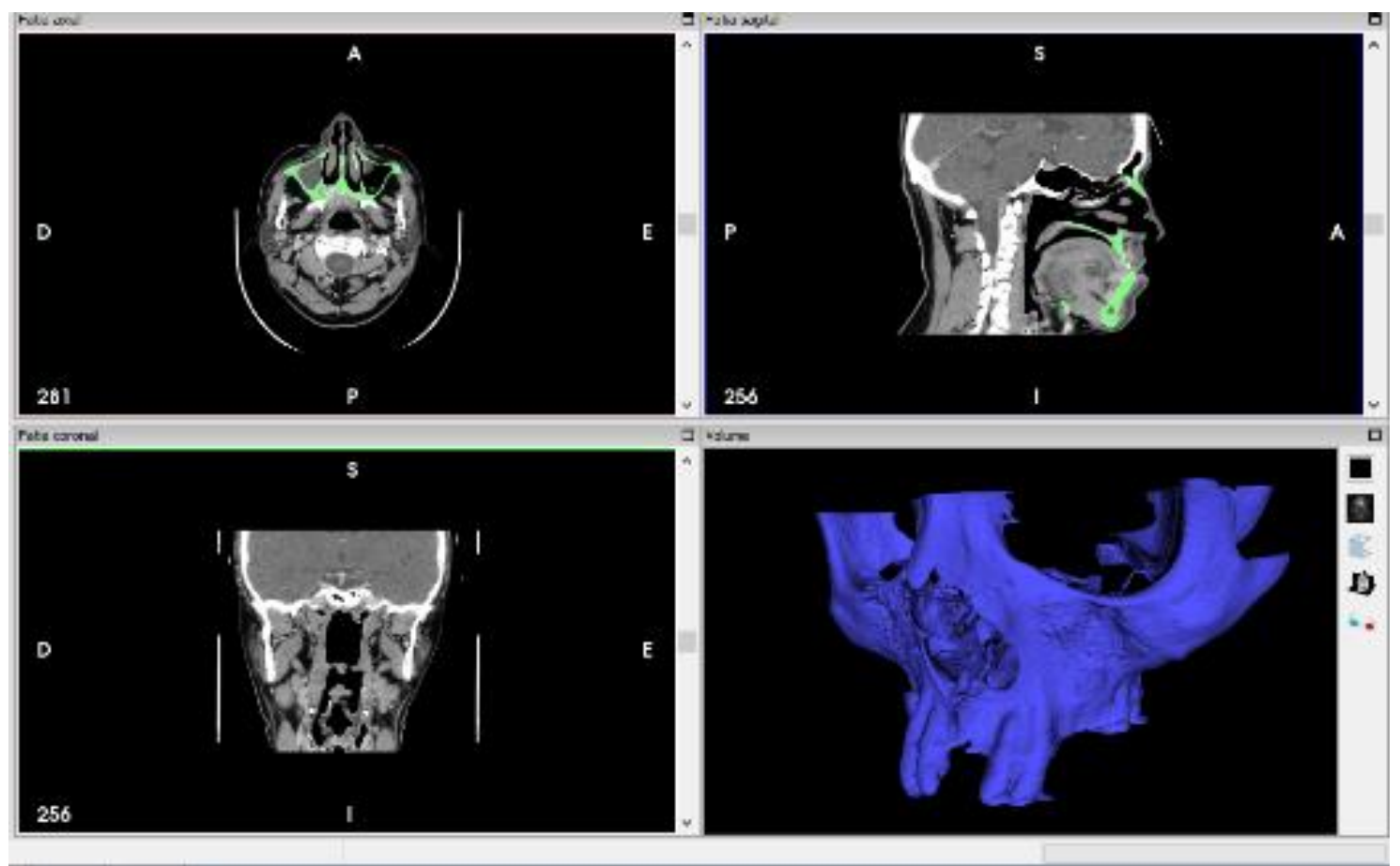

Figura 3: Posições Anatômicas da Região em Estudo.

\section{Tratamento da Imagem Médica} biomodelo.

Nessa etapa utilizou-se um software comercial CAD para reconstrução

As partes perdidas do paciente, Figura 4, foram reconstruídas com o auxílio dessa plataforma CAD, Figura 5. Pelo fato de não haver condições de duplicação ou reconstruções, aproveitando o mesmo órgão do paciente, foi utilizado um banco de dados de tomografias computadorizada de pacientes anônimos, fornecido pela Liga Norte Rio Grandense Contra o Câncer, onde pode-se verificar a compatibilidade das características físicas, anatômicas e raciais do paciente anônimo que melhor se adeque e adapte ao Paciente 02.

Uma vez que o modelo foi reconstruído tridimensionalmente, Figura 6, há a necessidade de converter a imagem tridimensional gerada para um formato que seja compreensível pelo sistema de prototipagem rápida. O formato STL é aceito como padrão e é o mais utilizado como interface entre os processos de prototipagem rápida. Sendo este, o utilizado nesta metodologia. Este processo será realizado com o auxílio da plataforma CAD. 


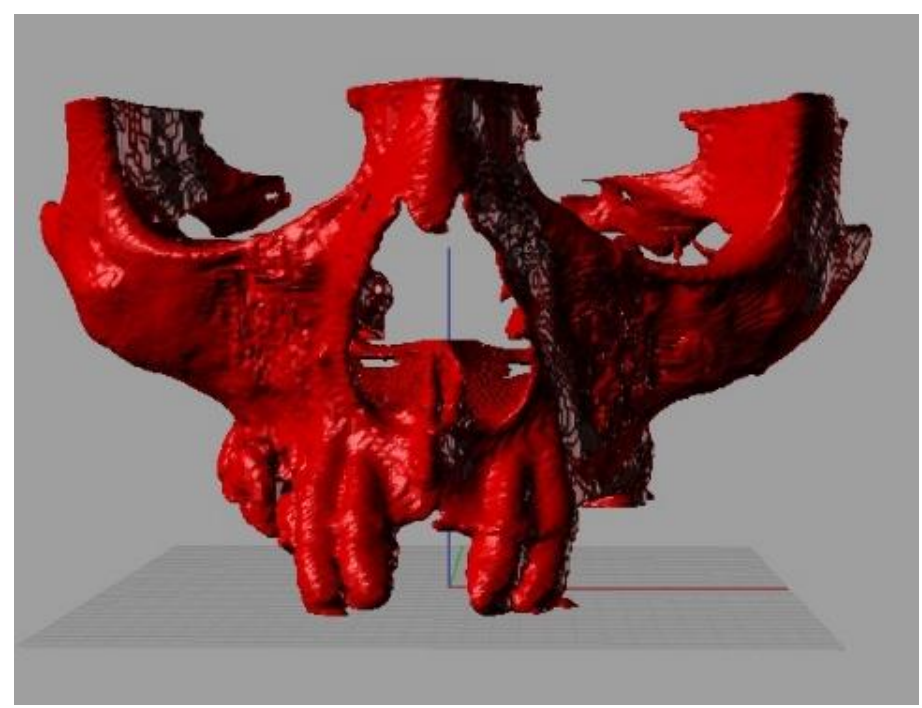

Figura 4: Modelo Inicial - Paciente 01.

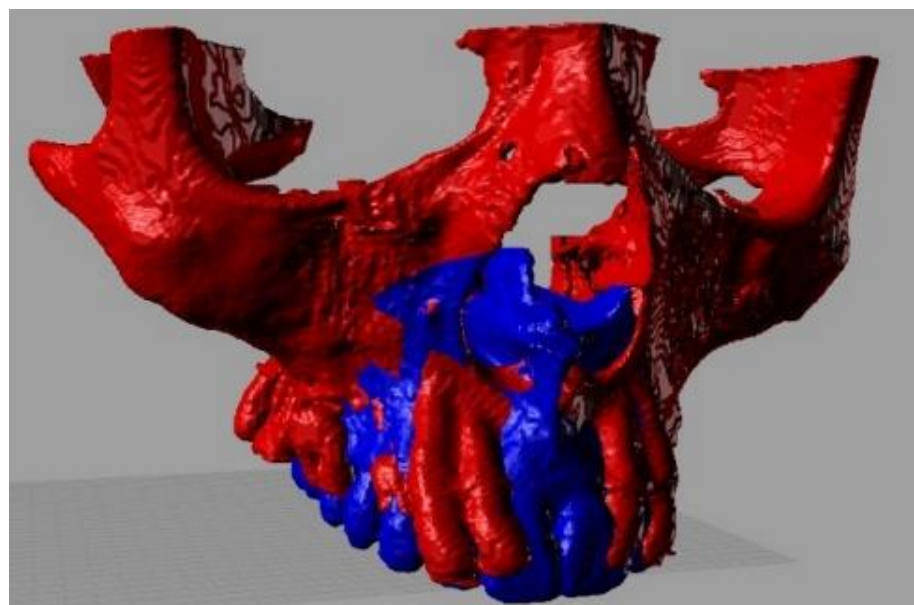

Figura 5: Reconstrução da Superfície Perdida - Paciente 01.

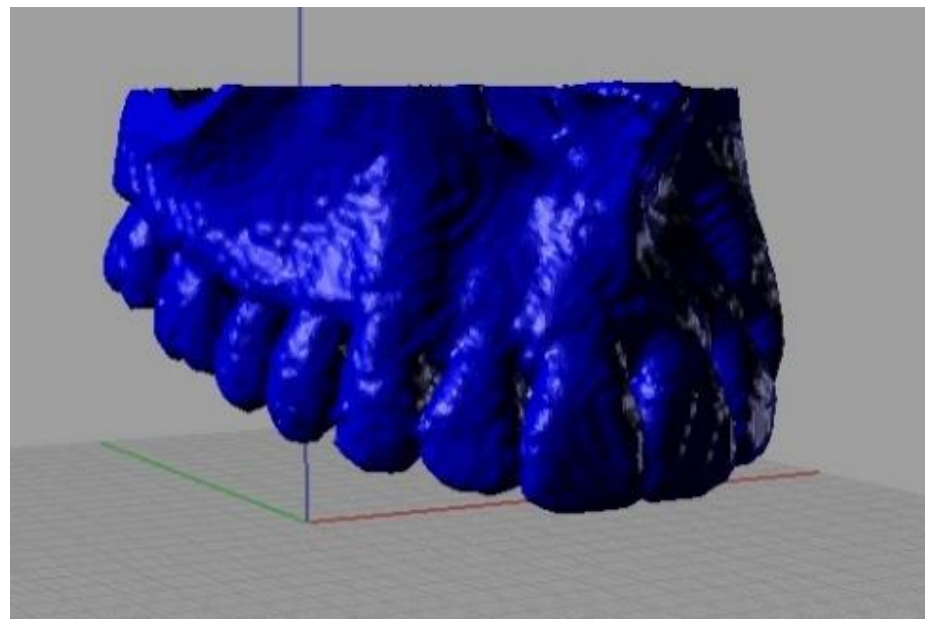

Figura 6: Modelo Final da Prótese- Paciente 02. 


\section{Confecção do Biomodelo}

A prototipagem rápida possibilita a confecção de modelos tridimensionais obtidos através de um modelo CAD trabalhado tridimensionalmente, ou através de imagens que foram obtidas através de scanners tridimensionais. Isto proporciona a criação de modelos reais a partir de modelos virtuais.

A manufatura em prototipagem rápida refere-se aos processos que constrói partes (camada por camada) de forma aditiva. A prototipagem rápida é muito utilizada em processos de manufatura na indústria automotiva, aeroespacial, telecomunicações e máquinas industriais. No entanto, outras possibilidades de aplicação estão emergindo, entre as quais o uso do processo de prototipagem rápida aplicada à área médica.

No processo de prototipagem rápida deste trabalho, o biomodelo foi construído em camadas, iniciando pela camada mais baixa e assim sucessivamente, construindo o objeto através de pilhas de camadas, até que se forme o objeto final. Esses sistemas são bastante precisos, de modo que as camadas podem ter espessuras de $0,1 \mathrm{~mm}$. Finalizado o processo de confecção, a prótese foi repassada para a equipe Clínica efetuar os respectivos procedimentos de limpeza e instalação no paciente.

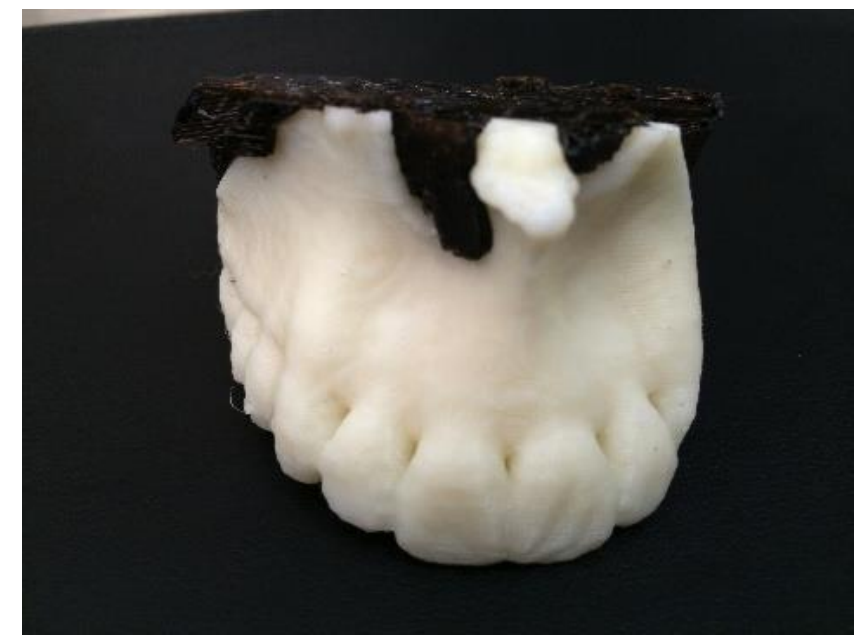

Figura 6: Prótese Fabricada por Prototipagem 3D - Paciente 01.

\section{RESULTADOS E DISCUSSÕES}

A prototipagem rápida $(\mathrm{PR})$ tem vindo afirmando-se como uma tecnologia de grande relevância no setor da saúde devido às suas enormes potencialidades no auxílio de procedimentos cirúrgicos complexos, moldes para guias cirúrgicos, planeamento de tratamentos, visualização de alguma estrutura anatômica específica, fabricação de próteses, tratamento de tumores, planeamento de radioterapia, diagnósticos, projeto de implantes, instrumentos médicos e outras aplicações.

Com este trabalho foi possível verificar que é possível realizar a prototipagem dos modelos, já que o arquivo STL foi gerado, sem falhas.

A modelagem das superfícies e sua transformação em modelo sólido permitirá uma futura análise por elementos finitos simulando as cargas e os efeitos causados.

A modelagem das superfícies demonstrou que é possível a obtenção de geometrias a partir de tecidos diferentes do ósseo, com o Software InVersalius. A superfície gerada apresentou-se apta, conforme esperado. 


\section{CONCLUSÃO}

Modelos físicos derivados de dados de (TC) ou (RM) podem oferecer aos médicos, cirurgiões e dentistas uma relação direta, compreensão intuitiva, de detalhes anatômicos complexos que não podem ser obtidos a partir de imagens.

$\mathrm{O}$ uso de prototipagem rápida associada a métodos de reconstrução tridimensional automáticas podem melhorar diversas áreas relacionadas a aplicações médicas, tais como a visualização $3 \mathrm{D}$ de uma anatomia específica, planejamento cirúrgico, projeto e fabricação do implante da prótese.

\section{AGRADECIMENTOS}

Agradecimentos especiais à Liga Norte-Riograndense Contra o Câncer/ Natal/ Brasil, ao Centro de Tecnologia da Informação Renato Archer/ Campinas/Brasil e ao LAIS - Laboratório de Inovação Tecnológica em Saúde da Universidade Federal do Rio Grande do Norte, localizado no Hospital Universitário Onofre Lopes/UFRN.

\section{REFERÊNCIAS}

[1] Arora, A., et al. Custom-Made Implant for Maxillofacial Defects Using Rapid Prototype Models, American Association of Oral and Maxillofacial Surgeons, Elsevier Inc, 2013.

[2] Choi, J.Y. et al. "Analysis of errors in medical rapid prototyping models", Int. J. Oral Maxillofac. Surg. 31: 23-32, 2002.

[3] Fernandes, A.U; Goiato, M.C; Dos Santos, D.M. Effect of weatheringand thickness on roughness of acrylic resin and ocular button. ContLensAnteriorEye. p. 33-124, Jun. 2010

[4] Foggiatto, J. A. O Uso Da Prototipagem Rápida Na Área Médico-Odontológica, UTFPR, 2010.

[5] Goiato, M.C; Fernandes, A.U; Dos Santos, D.M; Barão, V.A.R. Positioning Magnets on a Multiple/Sectional Maxillofacial Prosthesis. J Contemp Dent Pract. p. 101-107. 2007.

[6] Pedrini, H. Reconstrução 3d a partir de seções transversais de objetos. Dissertação de Mestrado - Departamento de Engenharia da Computação e Automação Industrial. Universidade Estadual de Campinas, Campinas, 98 p, 1994.

[7] Rezende, J.R.V. Fundamentos da prótese buco-maxilo-facial. São Paulo. Sarvier, 1987.

[8] Rodrigues, Shenoy e Shenoy; Prosthetic rehabilitation of a patient after partial rhinectomy: Aclinical report. J Prosthet Dent; p. 93-125. 2005.

[9] Safira. et al. Aplicação dos biomodelos de prototipagem rápida na Odontologia, confeccionados pela técnica da impressão tridimensional. Revista de Ciências Médicas e Biológicas. 2010.

[10] Sugar, A. et al. The development of a collaborative medical modeling service: organizational and technical considerations. Br. J. Oral Maxillofac. Surg., Edinburgh, v.42, p.323-330, 2004. 
[11] Vinagre, M. A. M. Obtenção de Modelos Anatómicos por Prototipagem Rápida, Dissertação de Mestrado, Universidade de Aveiro, 2008.

[12] Yan Yongnian, et al. Rapid Prototyping and ManufacturingTechnology: Principle, Representative Technics, Applications, and Development Trends, Tsinghua Science and Technology, ISSN 1007-0214 01/38, pp1-12, volume 14, number s1, 2009.

[13] Zhang, S., et al. Application of Rapid Prototyping for Temporomandibular Joint Reconstruction, American Association of Oral and Maxillofacial Surgeons. Elsevier Inc. All rights reserved, 2011.

[14] http://www.ligacontraocancer.com.br/ acesso dia 21 de março de 2013.

[15] Ariani N, Visser A, van Oort RP, Kusdhany L, Rahardjo TB, Krom BP, van der Mei HC, Vissink A. Current state of craniofacial prosthetic rehabilitation. Int J Prosthodont 2103;26:57-67.

[16] Flood TR, Russell K. Reconstruction of nasal defects with implant-retained nasal prostheses. Br J Oral Maxillofac Surg 1998;36:341-345.

[17] Huber H, Studer SP. Materials and techniques in maxillofacial prosthodontic rehabilitation. Oral Maxillofac Surg Clin North Am 2002;4:73-93.

[18] Wallace CG, Wei FC. The status, evolution and future of facial reconstruction. Chang Gung Med J 2008;31:441-449.

[19] Beumer J, Reisberg DJ, Marunick MT, Powers J, Kiat-amnuay S, van Oort R, Zhao Y, Wu G, Eversole LR, Cherrick HM, Roumanas E, Pedroche D, Baba T, de Cubber J, Moy PK, Noorda WD, van Dijk G. Rehabilitation of facial defects. In: Beumer J, Marunick MT,Esposito SJ editors. Maxillofacial rehabilitation: prosthodontics and surgical management of cancer-related, acquired, and congenital defects of the head and neck. 3d edition. Chicago: Quintessence Pubishing Co. 2011. p. 255-314.

[20] Mantri S, Khan Z. Prosthodontic rehabilitation of acquired facial defects. In: Agulnik M, editor: Head and neck Cancer. InTech 2012. p. 315-336.

[21] Chalian VA. Treating the patient with facial defects. In: Laney WR, editor. Maxillofacial prosthetics. Littleton, Massachusetts: PSG Publishing Co.; 1979. p. 279-308.

[22] Lemon JC, Chambers MS, Jacobsen ML, Powers JM. Color stability of facial prostheses. J Prosthet Dent 1995;74:613-618.

[23] Parel SM, Tjellström A. The United States and Swedish experience with osseointegration and facial prostheses. Int J Oral Maxillofac Implants 1991;6:75-79.

[24] Ethunandan M, Downie I, Flood T. Implantretained nasal prosthesis for reconstruction of large rhinectomy defects: the Salisbury experience. Int J Oral Maxillofac Surg 2010;39:343-349.

[25] Leonardi A, Buonaccorsi S, Pellachia V, Moricca LM, Indrizzi E, Fini G. Maxillofacial prosthetic rehabilitation using extraoral implants. J Craniofac Surg 2008;19:398-405.

[26] Mantri S, Khan Z. Prosthodontic rehabilitation of acquired facial defects. In: Agulnik M, editor: Head and neck Cancer. InTech 2012. p. 315-336.

[27] Chua, C. K., Chou, S. M., Lin, S. C., Lee, S. T. and Saw, C. A. (2000), "Facial prosthetic model fabrication using rapid prototyping tools", Integrated Manufacturing Systems, Vol. 11 No. 1, pp. 42-53.

[28] Cheah, C. M., Chua, C. K., Tan, K. H. and Teo, C. K. (2003a), "Integration of laser surface digitizing with $\mathrm{CAD} / \mathrm{CAM}$ techniques for developing facial prostheses. 
Part 1: Design and fabrication of prosthesis replicas", International Journal of Prosthodontics, Vol. 16 No. 4, pp. 435-41.

[29] Cheah, C. M., Chua, C. K. and Tan, K. H. (2003b), "Integration of laser surface digitizing with CAD/CAM techniques for developing facial prostheses. Part 2: Development of molding techniques for casting prosthetic parts", International Journal of Prosthodontics, Vol. 16 No. 5, pp. 543-8.

[30] Chandra, A., Watson, J., Rowson, J. E., Holland, J., Harris, R. A. and Williams, D. J. (2005), "Application of rapid manufacturing techniques in support of maxillofacial treatment: evidence of the requirements of clinical application", Proceedings of the Institution of Mechanical Engineers, Part B: Journal of Engineering Manufacture, Vol. 219 No. 6, pp. 469-76. 